\title{
Period of Eclipsing Binary EPIC 201458798
}

\author{
IVAn Altunin ${ }^{1}$, Ryan Caputo ${ }^{1}$, And KalÉe Tock ${ }^{1}$ \\ ${ }^{1}$ Stanford Online High School, Stanford, California, United States \\ "Corresponding author: ialtunin@ohs.stanford.edu, ryanjc@ohs.stanford.edu
}

\begin{abstract}
In this study we observed and determined the period of the eclipsing binary star EPIC 201458798, finding that its period has not changed since it was last measured and published in the Kepler catalog in 2014. We did this by developing Python code to determine the period using two independent algorithms and partially automating the search for suitable comparison stars. Our system was imaged by the $0.4 \mathrm{~m}$ Las Cumbres Observatory telescopes in four filters. We analyzed six photometry types returned for each image from the Our Solar Siblings pipeline. Of these photometries, we found source extractor kron (sek) to be the most appropriate type for our $2 \times 2$ binned images of EPIC 201458798. Both of the period-finding algorithms we developed gave results that were not statistically different from the Kepler catalog period for the infrared, red, and visual filter images. The blue filter images were statistically different from the Kepler catalog period for one of our algorithms but not the other. ()(i) $\Theta 2020$ Astronomy Theory, Observations and Methods Journal
\end{abstract}

\begin{abstract}
Keywords: binaries: eclipsing - celestial mechanics - methods: data analysis - techniques: photometric

https://doi.org/10.32374/atom.2020.1.1
\end{abstract}

\section{INTRODUCTION}

A subset of binary star systems are known as eclipsing binaries (EBs). These systems are aligned such that the two orbiting stars eclipse each other relative to Earth's line of sight. This produces a change in the observed brightness of the system as a function of time. Although most EBs are so close together that they appear as a single source of light, this brightness change during their eclipses allows us to deduce their characteristics, including period and position on the Hertzsprung-Russell diagram (Southworth, 2012). This makes EBs a primary and direct source of information on star properties and stellar evolution.

In this study, we measure EPIC 201458798, a short period ( $\mathrm{p}<1$ day) EB, and confirm the Third Revision of the Kepler Eclipsing Binary Catalog's period for this system using our own period-finding algorithms and an independently acquired data set (Kirk, 2016). In the process, we examine some photometric considerations that arise when using $0.4 \mathrm{~m}$ LCO telescopes with $2 \times 2$ image binning.

\section{TARGET SELECTION}

We chose an EB that was visible during the early spring because that was when our study was conducted. The Kepler 1 campaign contained stars that were observable during the spring, so we searched the Kepler Catalog in this campaign (Kirk, 2016).

Furthermore, we narrowed our selection to detached EBs, excluding semi-attached and contact bi- 
naries. Since detached binaries are completely separate, they can yield more accurate estimates of relative masses and temperatures of the individual stars (Rozmus, 2010). When searching the Kepler catalog, we were looking for a target with a Morph between 0 and 0.5. The Morph metric was developed for the second release of Kepler data to classify EBs by the shape of the primary eclipse. A system with Morph c $<0.5$ is predominantly detached, a Morph of $0.5<$ $\mathrm{c}<0.7$ is semi-detached, and a Morph of $0.7<\mathrm{c}<$ 0.8 is an overcontact binary (Matijevic, 2012).

In addition, our target had to have a period less than 1.5 days both to avoid using excessive telescope time and because we wanted to collect sufficient images to calculate the period from our own observations within a few weeks. The target also had to be sufficiently bright for the ground-based $0.4 \mathrm{~m}$ LCO telescopes to observe it; a system with kmag less than 13 was optimal. Finally, the eclipses needed to be deep enough to be accessible for analysis. A primary eclipse depth that was at 0.8 or deeper on a plot of normalized flux was deemed adequate.

\section{OUR TARGET}

Applying the criteria specified above, we settled on EPIC 201458798 as our target star. This system's characteristics are shown in Table 1 . The target star has a magnitude (kmag) close to 12 and a primary eclipse that causes its brightness to dip to 0.75 on a plot of normalized flux versus phase. Its lightcurve from the Kepler Eclipsing Binary Catalog, Third Revision, is shown in Figure 1 (Kirk, 2016).

Table 1. Characteristics of EPIC201458798

\begin{tabular}{cc}
\hline Property & Value \\
\hline RA (deg) & 168.2801 \\
Dec (deg) & -0.0925 \\
kmag & 12.091 \\
Period (days) & 0.6193964 \\
Period Error & 0.0000347 \\
Morph & 0.13 \\
\hline
\end{tabular}

\section{OBSERVATION HISTORY}

Our target was observed by Kepler from June 2, 2014 to August 21, 2014. These dates were found by con-

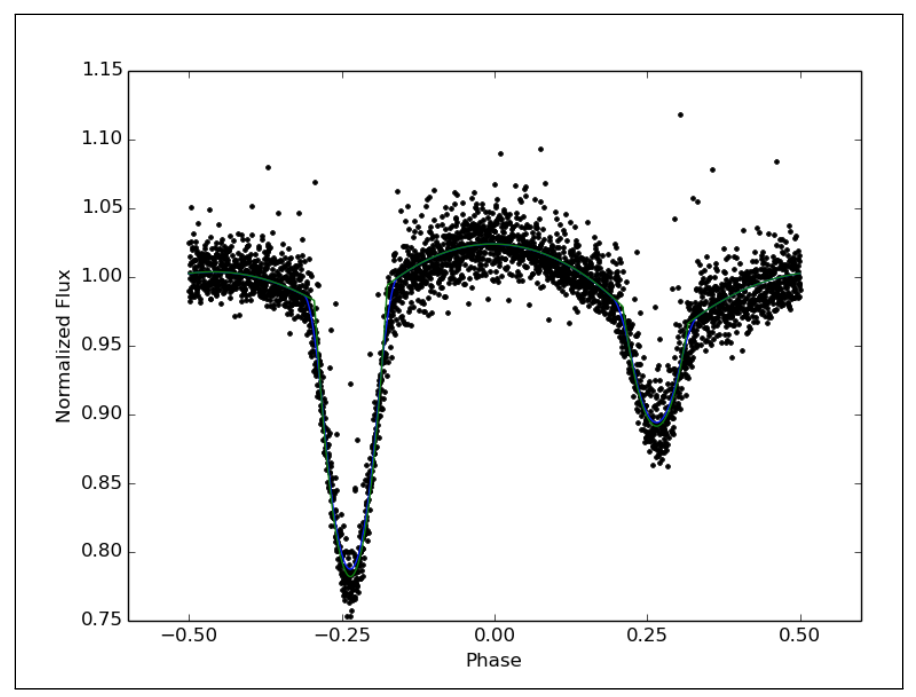

Fig. 1. EPIC 201458798 light curve from the Kepler Eclipsing Binary Catalog, Third Revision.

verting the modified Julian dates of the observations listed under "LC data" on the Kepler catalog website to the calendar date. In 2016, our target was part of the study "Planet Hunters K2" which aimed to detect exoplanets orbiting eclipsing binary stars. This study collected data on 75 targets from Kepler campaigns 1-3 using speckle interferometry on the Southern Astrophysical Research telescope and adaptive optics imaging on the Keck II telescope. Our target was not found to have exoplanets (Schmitt, 2016).

\section{INSTRUMENTS USED}

The telescopes used for our observations were part of the Las Cumbres Observatory network (Brown, 2013). All ten of these 0.4-meter telescopes have identical specifications. They are located at Siding Spring Observatory in Australia, South African Astronomical Observatory in Sutherland, South Africa, Cerro Tololo Interamerican Observatory in Chile, Teide Observatory in Spain, McDonald Observatory in Texas, United States, and Haleakala Observatory in Hawaii. All have an SBIG STL-6303 camera. A sample telescope is shown in Figure 2. Table 2 gives a count of the number of images of EPIC 201458798 that were returned by each of the telescopes.

\section{FILTERS}

Our images were taken in the Bessel B and V, Sloan Digital Sky Survey (SDSS) $r^{\prime}$ and i' filters, corresponding to blue, visible, red, and infrared light. A rough, handrawn sketch of the bandwidth comparison of 
Table 2. LCO telescope images of EPIC 201458798

\begin{tabular}{ccc}
\hline Telescope & Telescope ID & Number of Images \\
\hline Sliding Spring, New South Wales, Australia & $\mathrm{kb97,kb98}$ & 324 \\
SAAO, Sutherland, South Africa & $\mathrm{kb96}$ & 100 \\
Teide Observatory, Tenerife, Spain & $\mathrm{kb} 88, \mathrm{~kb} 99$ & 119 \\
CTIO, Region IV, Chile, Spain & $\mathrm{kb} 26, \mathrm{~kb} 95, \mathrm{~kb} 81$ & 313 \\
McDonald Observatory, Texas, USA & $\mathrm{kb80}$ & 39 \\
Haleakala Observatory, Maui, USA & $\mathrm{kb} 27, \mathrm{~kb} 82$ & 58 \\
Total & & 953 \\
\hline
\end{tabular}

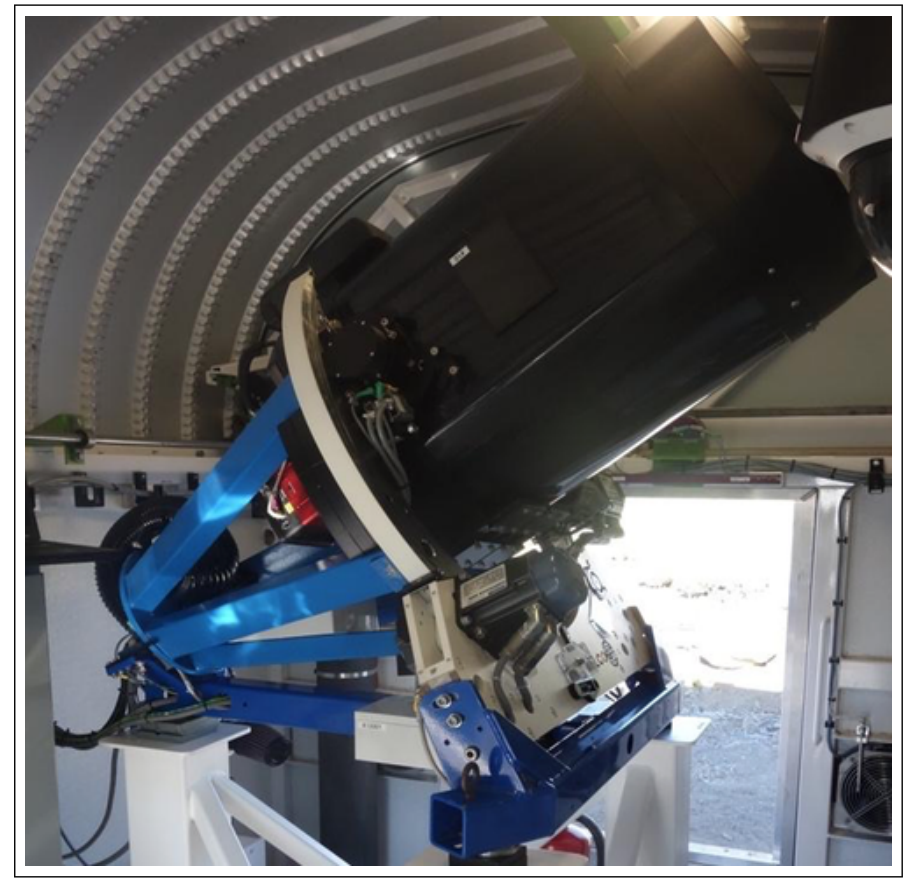

Fig. 2. A LCO 0.4m telescope. these filters to that of Kepler is shown in Figure 3 (Barentsen 2018), (Abazajian, 2009), (Giallongo, 2008).

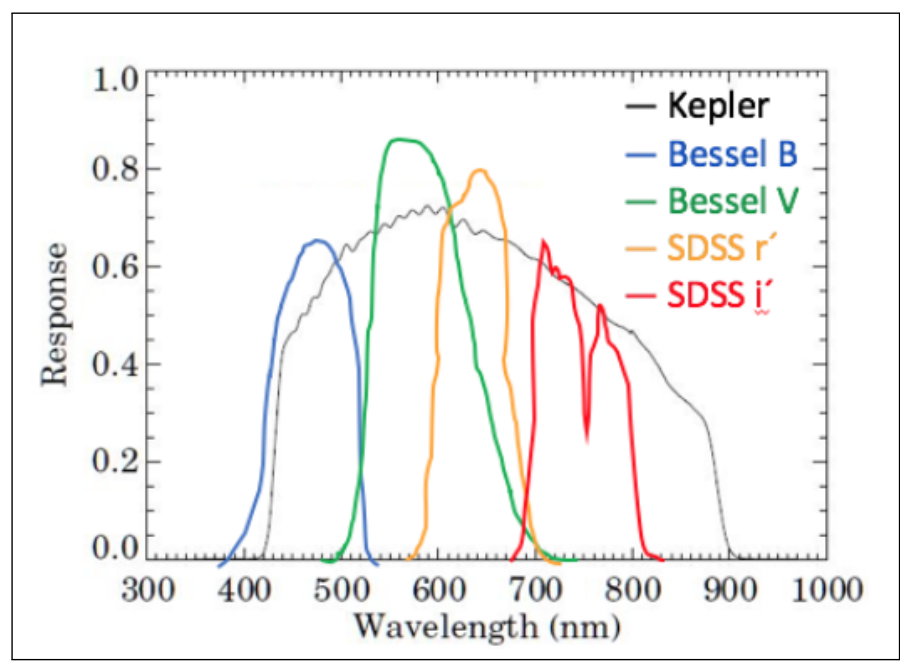

Fig. 3. Photometric response function of Kepler telescope compared to that of the filters used, sketched by hand from images from the references above.

\section{EXPOSURE TIMES}

The optimal exposure time in each filter was determined from test images of the target. Using AstroImageJ software, the ADU counts within an aperture surrounding the target are summed. The average ADU counts per pixel from the surrounding sky in an outer annulus is multiplied by the number of pixels within the target aperture and subtracted from this sum to obtain the AstroImageJ "Source minus Sky" number. Figure 4 shows a zoomed in image of the target in AstroImageJ surrounded by a 6-pixel radius aperture, which is in turn enclosed by the 8-pixel radius outer annulus used for the "Sky" counts. Because 
of the $2 x 2$ binning on the images used for this study, each pixel in Figure 4 actually represents 4 pixels worth of ADU counts.

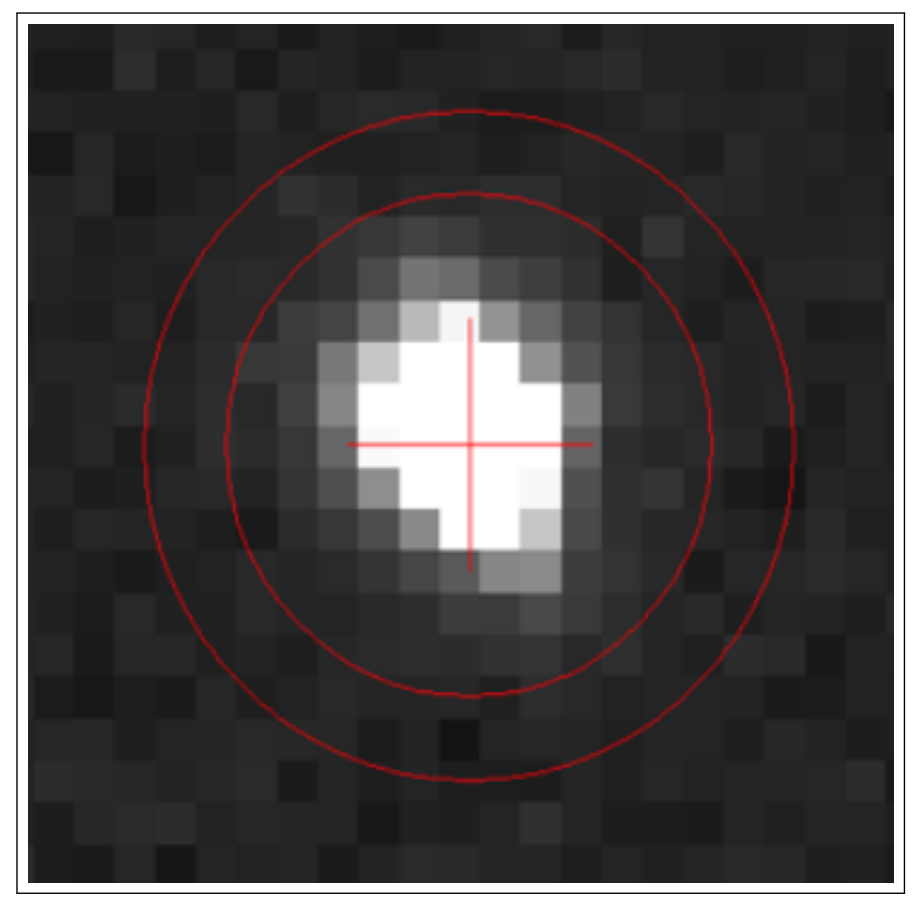

Fig. 4. Aperture photometry in AstroImageJ, using a 6-pixel radius target aperture and an outer annulus with an 8-pixel radius.

For a star like that shown in Figure 4, the ideal value of "Source minus Sky" is around 100,000 counts. A value much lower than 50,000 counts is associated with measurements that start to become dominated by noise. A value higher than 500,000 counts risks saturating the CCD camera on the telescope (Fitzgerald, 2018a). Complicating this is the fact that we expect our target star's brightness to vary (that is exactly what we are trying to measure!), possibly by a factor of 2 or more. Therefore, it is necessary not only for our test images' counts to be in range, but also double or half of those counts to be in range as well. Our initial 90s-exposure test images for the blue filter had photometry counts that were slightly on the low side (around 70,000 counts), so we increased the exposure time to 150 seconds for that filter. All of the other filters' test image exposure times were initially in range.

After the images were returned, we flipped through them by hand, scoring them from 0 (bad) to 4 (good) based on their quality. Small streaks (as from cosmic rays) that did not seem to affect the stars' images significantly earned a score of 3 . Slightly oval stars got a score of 2 , and very grainy or otherwise compromised images got a score of 1 . Images that were of the wrong starfield, or that were completely clouded over, or that lacked a plate solution due to a World Coordinate System registration failure got a score of 0 . In the final analysis, we used stars with scores of 2 or above. This represented a little more than half of the images returned, as shown in Table 3.

Table 3. Exposure times and image counts for the filters used.

\begin{tabular}{cccc}
\hline Filter & $\begin{array}{c}\text { Exposure } \\
\text { Time } \\
\text { (seconds) }\end{array}$ & $\begin{array}{c}\text { Total } \\
\text { Images } \\
\text { Returned }\end{array}$ & $\begin{array}{c}\text { Images } \\
\text { Used }\end{array}$ \\
\hline Bessel B & 150 & 226 & 121 \\
& 90 & 16 & 10 \\
SDSS i' & 45 & 239 & 124 \\
SDSS r $^{\prime}$ & 30 & 235 & 157 \\
Bessel V & 45 & 237 & 162 \\
\hline
\end{tabular}

\section{IMAGE REQUESTS: CADENCE AND JITTER}

We initially requested images to be taken with a cadence of approximately every couple of hours. LCO has a robotic scheduling system that recalculates which requests should be filled every 10 minutes based on priority, time sensitivity of the request, telescope availability, and weather (Pickles, 2014). Therefore, we knew that not all of our requests would be filled. EPIC 201458798 is eclipsing over approximately a third of its phase, so we initially set the "jitter" to be high (meaning that we gave the scheduler a broad time window in which to fulfill the request). Towards the end of the project, however, we were requesting images to be taken at specific times with lower jitter to fill in the primary eclipse. For these image requests, we calculated when the next eclipse would occur by taking the image date for the minimum flux in the lightcurve, and adding time in increments of one period until the present day.

\section{PHOTOMETRY}

The OSS pipeline (Fitzgerald, 2018b) performs six types of photometry on images that are returned from LCO: aperture photometry (Laher et al., 2012), source extractor photometry and source extractor kron photometry (Bertin \& Arnouts, 2018), and 
three types of point-spread function (PSF) photometry known as DAOPhot (Stetson, 1987), DOPhot (Schechter \& Mateo, 1993), and PSFEx (Bertin \& Arnouts, 2018). Aperture photometry is the simplest of these and is similar to the algorithm that is used by AstroImageJ to obtain the ADU counts referenced above. The photometry types will be referred to as apt, sex, sek, dao, dop, and psf, respectively. Sex is similar to apt, although it uses a different algorithm to undertake the aperture photometry. Sek also uses a similar algorithm except that the star's image is modeled as an ellipse rather than a circle, the size of which is varied to capture $90 \%$ of the object's light. The other three photometry types (dao, dop, and psx) report counts not by summing them but instead by fitting the counts for each pixel to various mathematical models of the way in which a star's brightness is expected to vary from pixel to pixel on an image.

The raw data returned by the OSS pipeline consists of text files containing the RA's and Dec's of the star centroids located by each photometry type in each image, along with their associated pixel coordinates, integrated ADU counts, and count errors. To find the counts for our target, we wrote Python code to scan through the RA's and Dec's of the image text files, finding the closest one based on its coordinates. As is evident in Figure 4, the target's light is smeared out over a diameter of about 7 pixels. Since the pixel scale for our images is approximately $1.16^{\prime \prime} /$ pixel, this amounts to about $8^{\prime \prime}$. We identified the target in each image as the star whose computed centroid fell within $2^{\prime \prime}$ of its expected coordinates from Table 1. This is comfortably within the image of the star and also corresponds to the amount that the star's apparent coordinates might vary with slight changes in the air's index of refraction due to passing cells of warm or cold air (the "seeing").

\section{COMPARISON STARS}

In addition to the target, it is necessary to observe a few other stars in the same field of view. These are denoted as "comparison stars" or just "comp stars". These stars enable our observation to be calibrated against atmospheric fluctuations and differing exposure times, as they provide a relative measurement of brightness rather than an absolute one. To make this relative measurement, the ratio between the intensity of the target and the comp star is taken. This is equivalent to the difference of the star magnitudes, where magnitude is the logarithm of intensity. However, this only works if the comp stars are not intrinsically variable themselves.

The first criteria for choosing comp stars for EPIC 201458798 was that they needed to be in the $27.5^{\prime} \mathrm{x}$ $18.5^{\prime}$ field of view surrounding the target. In addition, they had to be roughly the same color as the target star to keep everything as similar as possible. The comp stars also needed to be round in shape. This is a rule of thumb to verify that they are exposed properly and that they are single stars.

Figure 5 shows the star field, with our target star labeled along with six potential comp stars, located by examining a returned LCO image and also inspecting the corresponding starfield in AladinLite software (Boch \& Fernique, 2014). The particularly bright star at the top of the image is a cause for concern as it might induce some error in the photon counts for stars nearby. Table 4 shows the coordinates and magnitudes of the stars identified in Figure 5.

\section{LIGHTCURVE CONSTRUCTION}

The visually-identified comp star candidates in Table 4 were used to construct lightcurves of the target star for each of the six photometry types. Due to the short period of the target EB, our observations span several periods. Therefore, plotting the target-minus-comp magnitude versus Julian date results in a visually apparent random scatter of data, as shown at the left in Figure 6. Although it is clear that the images were requested in four separate batches spaced out over a few months, the lightcurve itself is not discernible when plotted versus date. To organize this data, it must be "phased", or plotted as a function of relative phase over the course of a single period. The phase of an observation is equal to the relative Julian date (observation date in days minus initial observation date in days) divided by the Kepler period and modulated by one. For example, if the Julian date of the first observation is 2458600.0 days and the period of the system is 3 days, then an observation taken on 2458601.5 days and an observations taken on 2458604.5 days would both have phase of 0.5 . As shown on the right in Figure 6, the target's primary and secondary eclipses become evident when the lightcurve is plotted in this way.

To normalize the lightcurve, the average flux of a region of the graph where the system is not eclipsing is set equal to 1 , and all other fluxes are plotted relative to that. For this system, the region of the graph where the system is not eclipsing is taken to be 


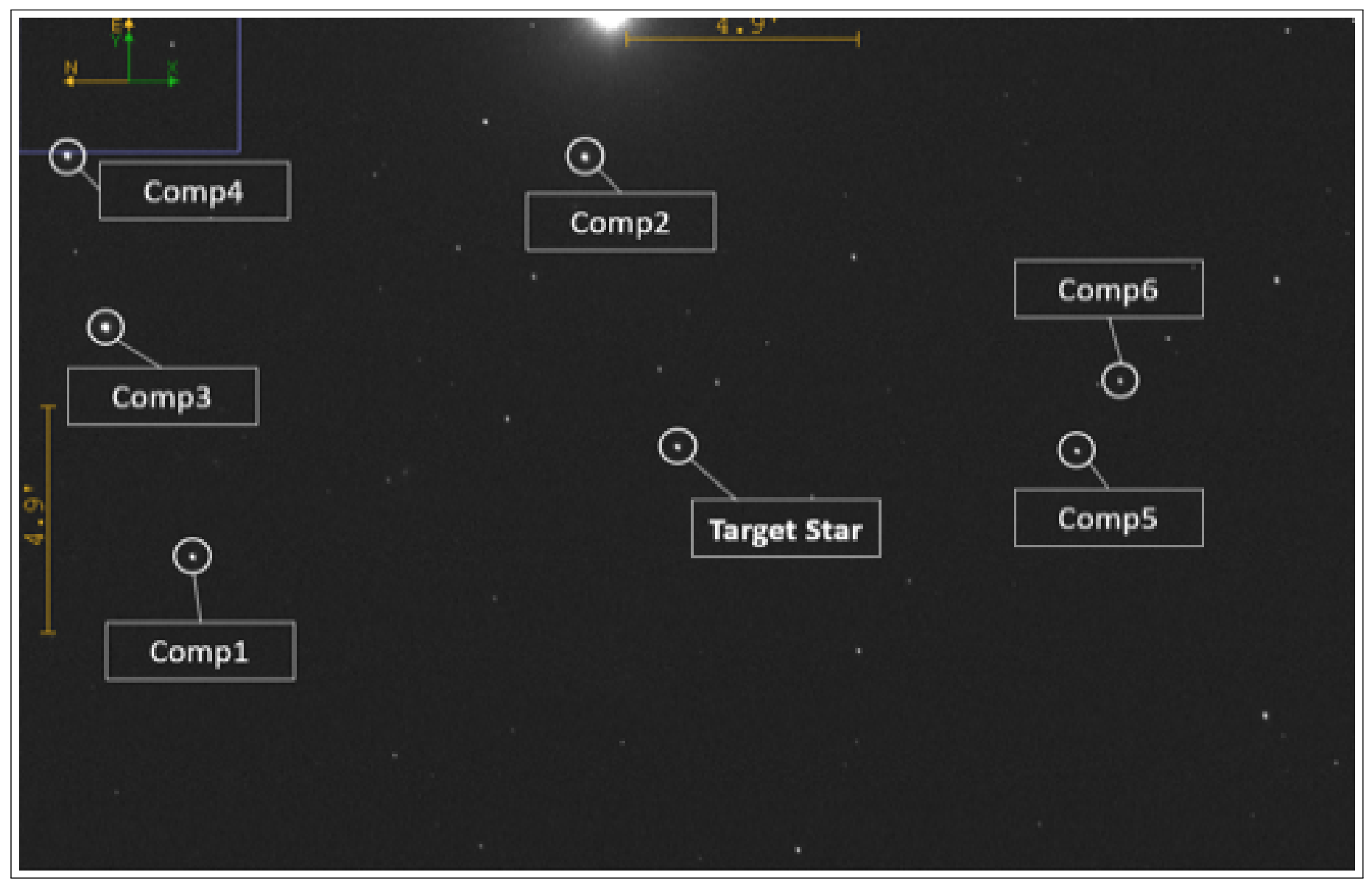

Fig. 5. Star field of EPIC 201458798, taken from one of our images.

Table 4. Stars in the field of view of EPIC 201458798.

\begin{tabular}{ccccc}
\hline & $\begin{array}{c}\text { Star } \\
\text { Identifier }\end{array}$ & $\begin{array}{c}\text { RA } \\
\text { HH:MM:SS }\end{array}$ & $\begin{array}{c}\text { Dec } \\
\text { Deg:min:sec }\end{array}$ & $\begin{array}{c}\text { Mag } \\
\text { B Filter }\end{array}$ \\
\hline Target & EPIC 201458798 & $11: 13: 07.231$ & $-00: 05: 33.01$ & 12.8 \\
\hline Comp 1 & TYC 263-777-1 & $11: 13: 07.231$ & $-00: 05: 33.01$ & 12.8 \\
\hline Comp 2 & $\begin{array}{c}\text { UCAC2 31687136 } \\
\text { High Proper Motion Star }\end{array}$ & $11: 13: 32.232$ & $-00: 03: 37.81$ & 11.841 \\
\hline Comp 3 & BD+00 2760 & $11: 13: 17.825$ & $+00: 06: 28.96$ & 10.552 \\
\hline Comp 4 & N/A & $11: 13: 32.626$ & $+00: 07: 15.85$ & 11.18 \\
\hline Comp 5 & N/A & $11: 13: 06.703$ & $-00: 13: 57.27$ & 13.58 \\
\hline Comp 6 & N/A & $11: 13: 12.724$ & $-00: 14: 51.80$ & 12.8 \\
\hline
\end{tabular}


between phase 0.3 and 0.4. On the Kepler Eclipsing Binary Catalog plot of Figure 1, the depth of the primary eclipse is approximately 0.75 , but for our data, the depth is closer to 0.65 and even lower for the blue filter (almost as low as 0.6). This likely stems primarily from differences between the filter on the Kepler telescope and the filters used here, whose response curves are sketched above in Figure 3. Also, some of the discrepancy may be due to a different choice of where the system is deemed to be out of eclipse. Finally, the primary eclipse depths can change in different filters due to the stars' unequal temperatures.

\section{CHOICE OF PHOTOMETRY}

Figure 7 shows the same lightcurve with all six types of photometry, using Comp 1 as the comparison star, and using the Kepler period to compute the phase. Based on the cleaner visual appearance of the lightcurve, we decided that sek photometry was most appropriate for images of this system taken with the $0.4 \mathrm{~m}$ LCO telescopes. However, it is noteworthy that the images used here had $2 \times 2$ binning, meaning that the integrated photometry count from sets of four adjacent pixels was reported. This type of binning has the advantages of a faster readout, a higher signal-to-noise ratio, and a smaller image filesize than $1 x 1$ binning, in which the photometry count of each pixel is recorded separately. However, in June of 2018, after this study was concluded, LCO switched to $1 \mathrm{x} 1$ binning. The increased spatial resolution of this method may cause different photometry types, particularly those that use PSF methods, to be more appropriate for future studies using the $0.4 \mathrm{~m} \mathrm{LCO}$ telescopes.

\section{COMPARISON STARS REVISITED WITH AUTO- MATED SELECTION}

Because they are interdependent, the choice of a comp star and selection of photometry is necessarily a somewhat cyclical process. The procedure described above for choosing provisional comp stars by eye, inspecting the resulting lightcurves, and selecting a photometry type must be followed by a re-examination of the comp stars using that photometry. So, after choosing sek as our photometric method, we wrote code to inspect the stars across all of the images to ensure that we were making the best possible choice of comp star for sek photometry.
For each star in each image, we counted how many sek-processed images contained that same star within $2^{\prime \prime}$ of RA and Dec. The coordinates were computed as those that enabled the star to be identified in the highest number of images. Any non-target star that occurred in more than $75 \%$ of the images with photometry counts between 40,000 and 500,000 was deemed as a potential comp. In this field of relatively dim stars, only 5 candidates met these criteria. Three of those were Comp Stars 1, 2, and 5 in the list of visually-identified comps from Table 4 . We decided to call the other two automatically-identified stars Comp 7 and Comp 8. They are shown in Figure 8.

To ensure that the comp stars were not themselves variable, all of their differential magnitudes were plotted versus each other. This resulted in 4 compversus-comp plots for each of the 5 candidates in 4 filters: a total of 80 plots, where 60 were unique (e.g. Comp1/Comp8 contained the same information as Comp 8/Comp1). Most of these showed flat horizontal lines, as shown in Figure 9.

However, Comp 2 had a slight curve when plotted versus all four other candidates. Although this eliminated it as a potential comp star for this study, its curve may cause Comp 2 to become interesting in its own right. If it is variable, it has an unknown period. To disentangle what if anything might be going on with this star, it would be necessary to continue monitoring it. Also and perhaps significantly, Comp 2 was the closest star to the bright star at the top of the star field.

To determine which of the other comp star candidates to use, we examined the remaining differential magnitude graphs, looking for the flattest lines. It was helpful to arrange the plots as a matrix in order to disentangle the effect of one comp star from that of another. For example, the curve shown for Comp 2 above should not eliminate the comp star against which it was plotted, because this feature showed up for all of the Comp 2 plots. In addition to examining the comp-versus-comp plots visually, we also compared the standard deviations of the differential magnitudes and the slopes from linear fits of each graph, took into account the roundness, color, and average counts from the comp star candidate compared to the target across all of our images, and compared the scatter of the phased lightcurve of the system plotted using sek photometry. So many competing factors made the selection difficult, but in the end, we chose Comp 1 as our comp star. 


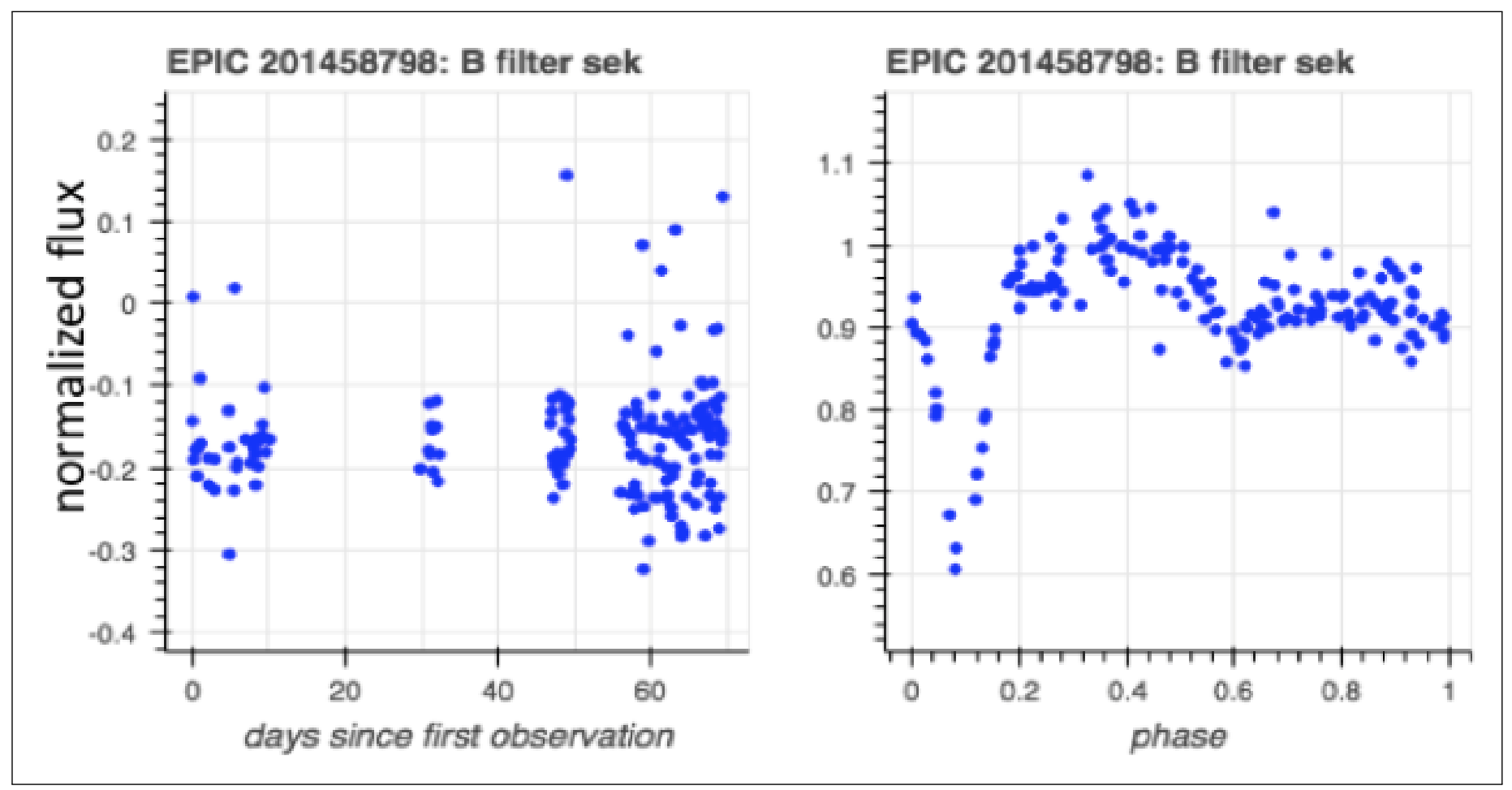

Fig. 6. Differential magnitude of EPIC 201458798 in the Bessel B filter as a function of date (left) and as a function of phase (right) using Comp 1, sek photometry, Kepler period.

\section{CALCULATING THE PERIOD}

To independently find the period of our EB, we guessed the period in iterations of one second and constructed the system's lightcurve based on that period. Since the period listed for this system on the Kepler site was 0.6193964 days, we checked periods within 0.4 days of that value. A correct period guess should cause the fluxes to arrange themselves into a recognizable lightcurve, as shown in Figure 10. However, visual examination of thousands of lightcurves generated by incriminating the period guess by one second was impractical and prone to subjectivity. Instead, an automated method was needed to determine which of the guessed periods was correct.

Two different phase dispersion minimization (PDM) algorithms were employed to determine the correctness of each period guess so that the results could be compared for consistency. Both algorithms operate under the assumption that if the guessed period for a system is correct, then two points with similar phase with also have similar flux, putting them relatively close together in flux-phase space. This is visually apparent in Figure 10. Within the tool, as the slider is moved to adjust the period parameter $\mathrm{p}$, the correctness of any given period can be assessed by how close the fluxes are for points that are adjacent in phase.

\section{PDM ALGORITHM 1: MINIMUM DISTANCE METHOD FOR FINDING THE PERIOD}

For the distance method, a period is guessed, and the distance between adjacent points on the resulting flux-versus-phase plot is computed using the distance formula. Once all the distances between adjacent points have been determined, these are summed, and the result is stored in a list. Another period is guessed, and the process is repeated. The minimum distance sum will correspond to a flux versus phase plot in which adjacent points are close to each other. For example, the plot on the right of Figure 10 has a smaller distance between adjacent points than the plot on the left. Therefore, we can conclude that the plot on the right's corresponding period is more likely to be the correct period for the system. (Dworetsky, 1983).

This process of guessing periods and computing the distance sums continues until the range of periods within \pm 0.4 days of the Kepler period is exhausted, iterating by 1 second. This range greatly exceeds the period changes typically observed for EBs, which are on the order of seconds per year (Lohr, 2012). Since the Kepler period was computed only four years 


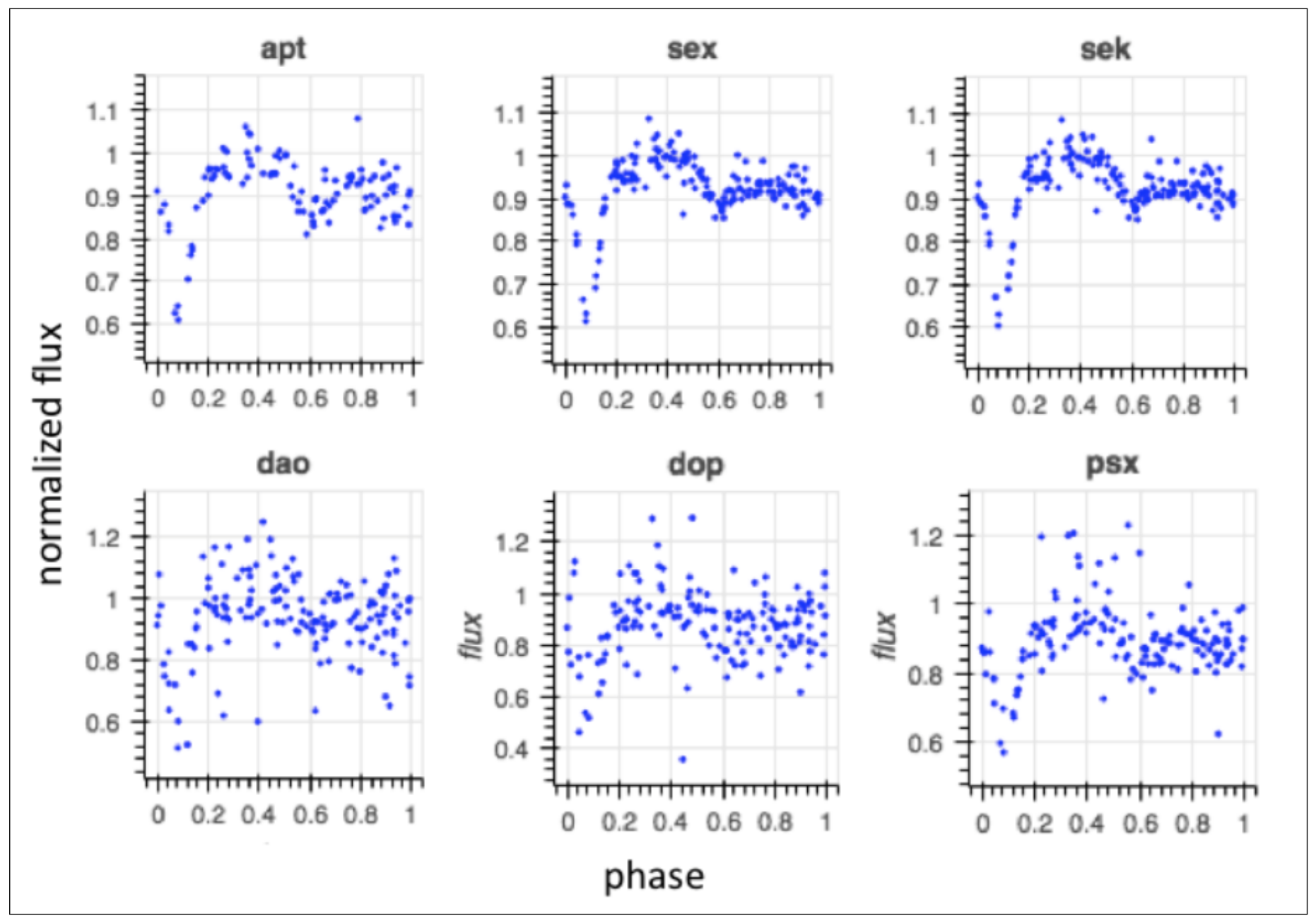

Fig. 7. Lightcurve in blue filter with various photometries, using Comp 1 as the comparison star, and using the Kepler period to compute the phase. 


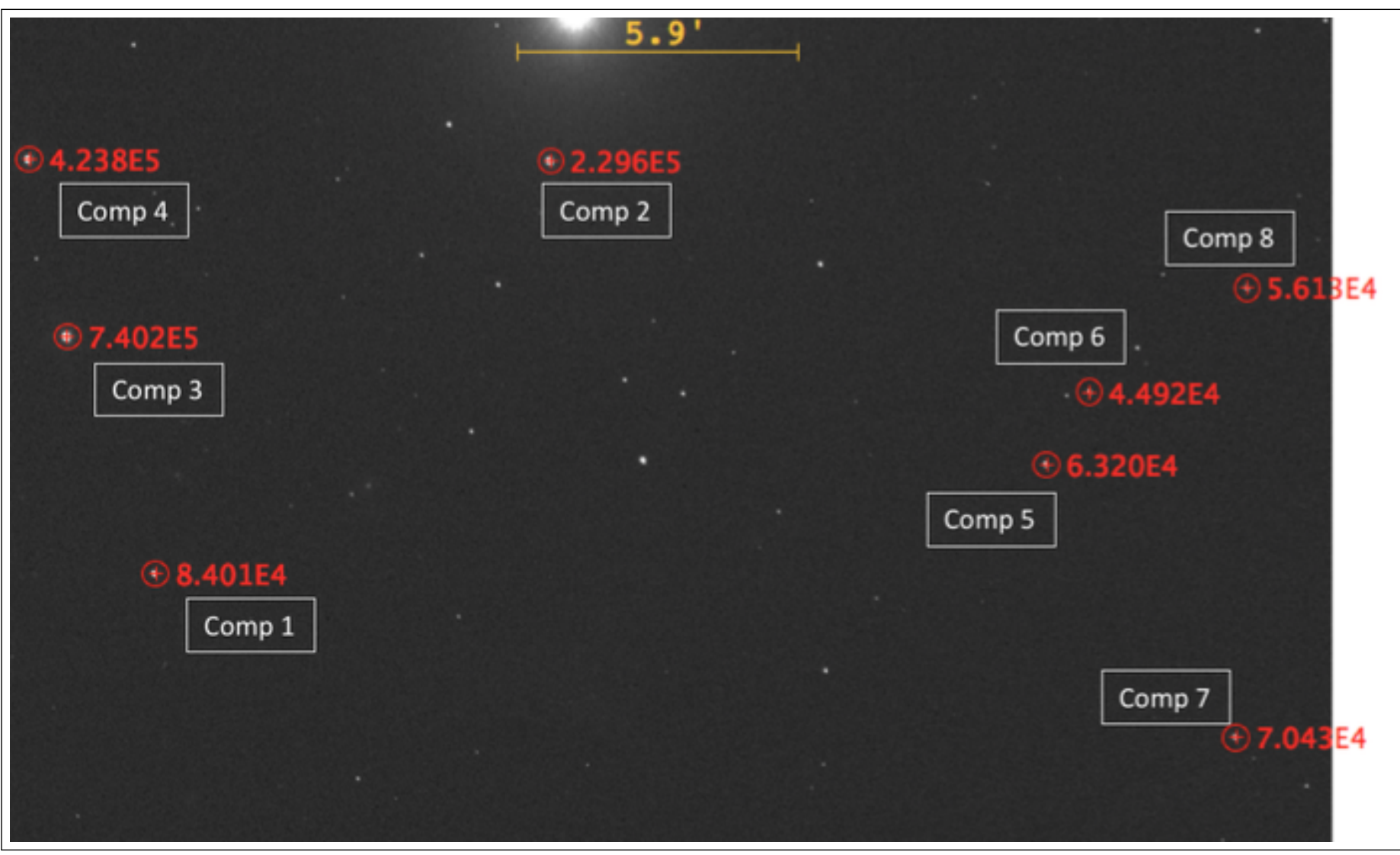

Fig. 8. EPIC 201458798 star field including the automatically-identified Comps 7 and 8 at right.

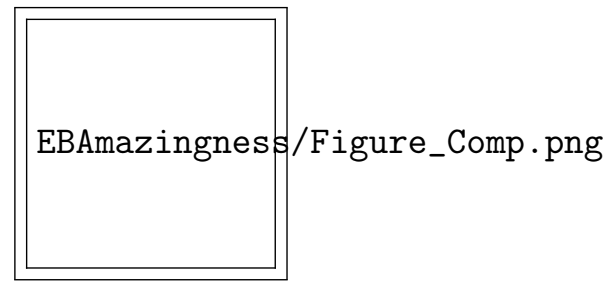

Fig. 9. A sample lightcurve for comp stars. All comp lightcurves are shown in the supplemental documents.

before this project, the period is not expected to have changed significantly in the interim.

\section{PDM ALGORITHM 2: MINIMUM STANDARD DE- VIATION METHOD FOR FINDING THE PERIOD}

For the standard deviation method, a period is guessed, and the resulting fluxes are sorted into bins based on phase, as shown in Figure 11.

The fluxes with a phase from 0 to 0.1 are in the first bin, 0.1 to 0.2 in the second, 0.2 to 0.3 in the third, and so on. The standard deviation of the fluxes is taken for each of the bins, the ten standard deviations are summed, and then stored in a list. As before, this process is repeated for every period within \pm 0.4 days of the Kepler period, with increments of 1 second. The period given by this method is the period guess that corresponds to the lowest standard deviation-sum (Stellingwerf, 1978). This is because the lowest sum indicates that the points within the phase bins of the light curves have similar fluxes.

\section{RESULTS}

The best periods found by the algorithms above are shown in Table 5, and the resulting lightcurves are plotted in Figure 12.

\section{ESTIMATING ERROR}

There is no universally accepted way of finding error for these types of algorithms, though many methods have been suggested (Montogomery \& Odonoghue, 1999). To obtain the error values shown in Table 5 , we used a method suggested by Michael Fitzgerald (Fitzgerald, 2018a). The points of the plot in Figure 13 represent the PDM result, shown on the vertical 


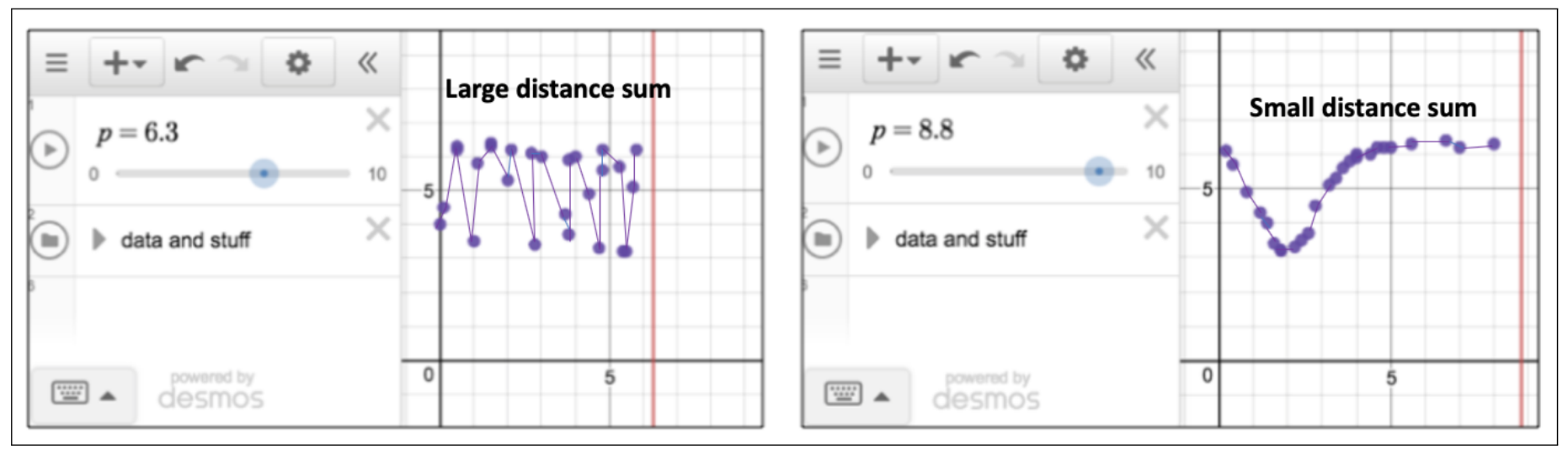

Fig. 10. Hensley's interactive Desmos tool demonstrating a more recognizable lightcurve and a smaller distance sum when fluxes (vertical axis) are plotted versus time over the course of one period for correct ( $\mathrm{p}=$ 8.8) versus incorrect $(\mathrm{p}=6.3)$ period (Hensley, n.d.).

Table 5. The best periods found by each algorithm for each filter for EPIC 201458798, compared to the Kepler period of 0.6193964 days (53516 seconds).

\begin{tabular}{ccccccc}
\hline Filter & $\begin{array}{c}\text { Minimum } \\
\text { distance best } \\
\text { period (s) }\end{array}$ & $\begin{array}{c}\text { Minimum } \\
\text { distance } \\
\text { best period } \\
\text { difference } \\
\text { from } \\
\text { Kepler (s) }\end{array}$ & $\begin{array}{c}\text { Error } \\
(\mathrm{s})\end{array}$ & $\begin{array}{c}\text { Minimum } \\
\text { standard } \\
\text { deviation } \\
\text { best period } \\
(\mathrm{s})\end{array}$ & $\begin{array}{c}\text { Minimum } \\
\text { standard } \\
\text { deviation best } \\
\text { period } \\
\text { difference } \\
\text { from Kepler } \\
(\mathrm{s})\end{array}$ & $\begin{array}{c}\text { Error } \\
\text { (s) }\end{array}$ \\
\hline Bessel B & 53491 & -25 & \pm 20 & 53516 & 0 & \pm 20 \\
\hline Bessel V & 53514 & -2 & \pm 8 & 53475 & -41 & \pm 45 \\
\hline SDSS r' & 53521 & 5 & \pm 6 & 53517 & 1 & \pm 12 \\
\hline SDSS i & 53515 & -1 & \pm 8 & 53518 & 2 & \pm 10 \\
\hline
\end{tabular}




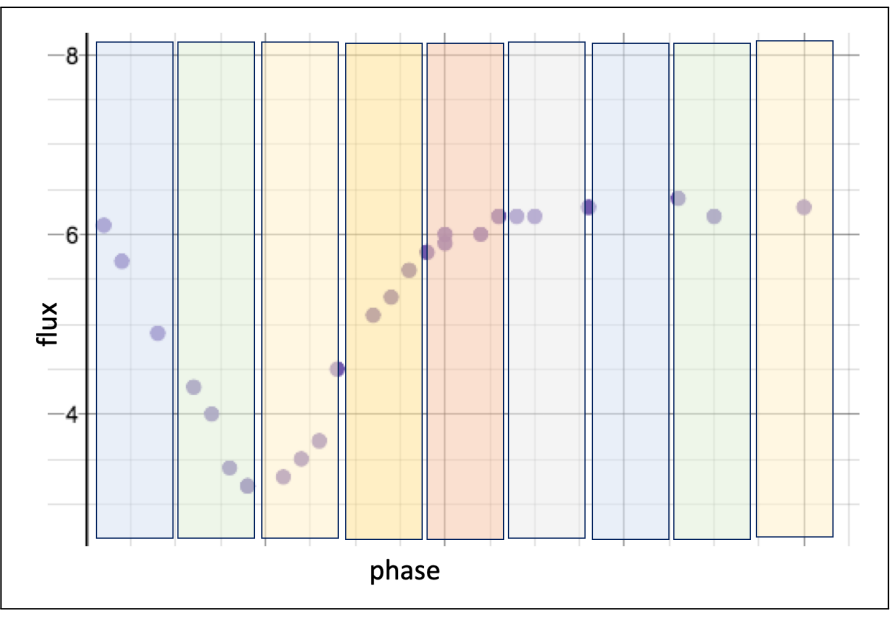

Fig. 11. Method for finding the best period from the sum of the standard deviations of the binned fluxes.

axis, as a function of period guess, which is shown on the horizontal axis. The period that corresponds to the lowest point on this plot is the period our algorithm returns. We estimate the error of this period as the width of the curve $5 \%$ of the way up from the minimum. This gives an approximation of the amount of scatter surrounding the period found by the algorithm. The error estimates of Table 5 were obtained visually by zooming in on the plot in the region of the minimum. As is evident from the numbers in the table, the period results that were the farthest from Kepler's showed the most evidence of scatter about the minimum and therefore had the highest error. The SDSS $r^{\prime}$ and $i^{\prime}$ filters had both the lowest error and the closest period results to the period calculated by Kepler.

The four filters and two algorithms used generated 8 PDM plots for sek photometry. As expected, secondary minima were observed at semi-regular intervals from the main minimum. For the red filter distance algorithm, but not for the other filters or for the standard deviation algorithm, the secondary minima occurred with an interesting pattern, shown in Figure 14.

\section{CONCLUSION}

We developed Python code to process time series data from the six types of photometry that are returned by the Our Solar Siblings pipeline, and to automate the search for appropriate comp stars within that data set. We found source extractor kron photometry to be the most appropriate of the six for our magnitude 12 eclipsing binary system imaged with the $0.4 \mathrm{~m} \mathrm{LCO}$ telescopes. We developed Python code to compute a period for EPIC 201458798 according to two different algorithms. Neither algorithm had a significant deviation from the Kepler period, indicating the period has not changed over the past four years since it was last measured by Kepler.

\section{ACKNOWLEDGMENTS}

This work has made use of data from the European Space Agency (ESA) mission (Gaia Website), processed by the Gaia Data Processing and Analysis Consortium (DPAC). Funding for the DPAC has been provided by national institutions, in particular the institutions participating in the Gaia Multilateral Agreement.

Special thanks to Michael Fitzgerald of Our Solar Siblings for his inspiration and generous and frequent support throughout this project.

Special thanks to Stanford Online High School for its support of student research and to Gary Oas for his help with Latex.

\section{SUPPLEMENTAL DOCUMENTS}

The images used for this analysis are here. The PDM Python code developed for this analysis is here. The comparison star analysis Python code, including all of the comp vs. comp stars plots is here.

\section{REFERENCES}

Abazajian, K. e. a. (2009). The seventh data release of the sloan digital sky survey. The Astrophysical Journal Supplement Series, 182(2).

Bertin, E., \& Arnouts, S. (2018). Sextractor: Software for source extraction. Astronomy and Astrophysics Supplement Series, 117(2), 393-404.

Boch, T., \& Fernique, P. (2014, May). Aladin Lite: Embed your Sky in the Browser. In N. Manset \& P. Forshay (Eds.), Astronomical data analysis software and systems xxiii (Vol. 485, p. 277).

Brown, T. M. e. a. (2013). Las cumbres observatory global telescope network. Publications of the Astronomical Society of the Pacific, 125(931), 1031-1055.

Dworetsky, M. M. (1983). A period-finding method for sparse randomly space observations or "how long is a piece of string?". Monthly Notices of the Royal Astronomical Society, 203(4), 917-924.

Fitzgerald, M. e. a. (2018a). Our solar siblings. a high school focused robotic telescope-based astron- 


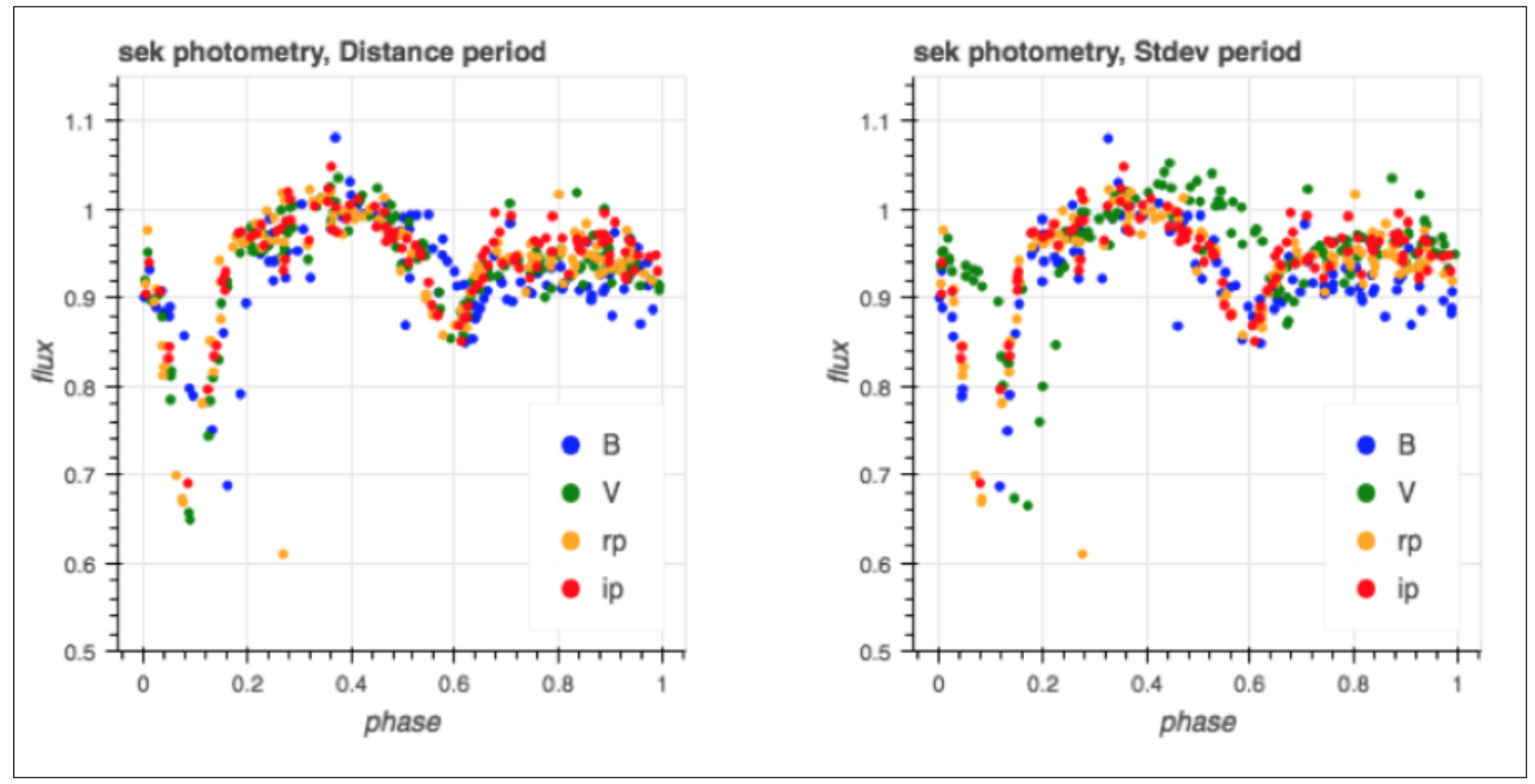

Fig. 12. Sek photometry lightcurves constructed from minimum distance method best periods for each filter (left) and minimum standard deviation periods for each filter (right).

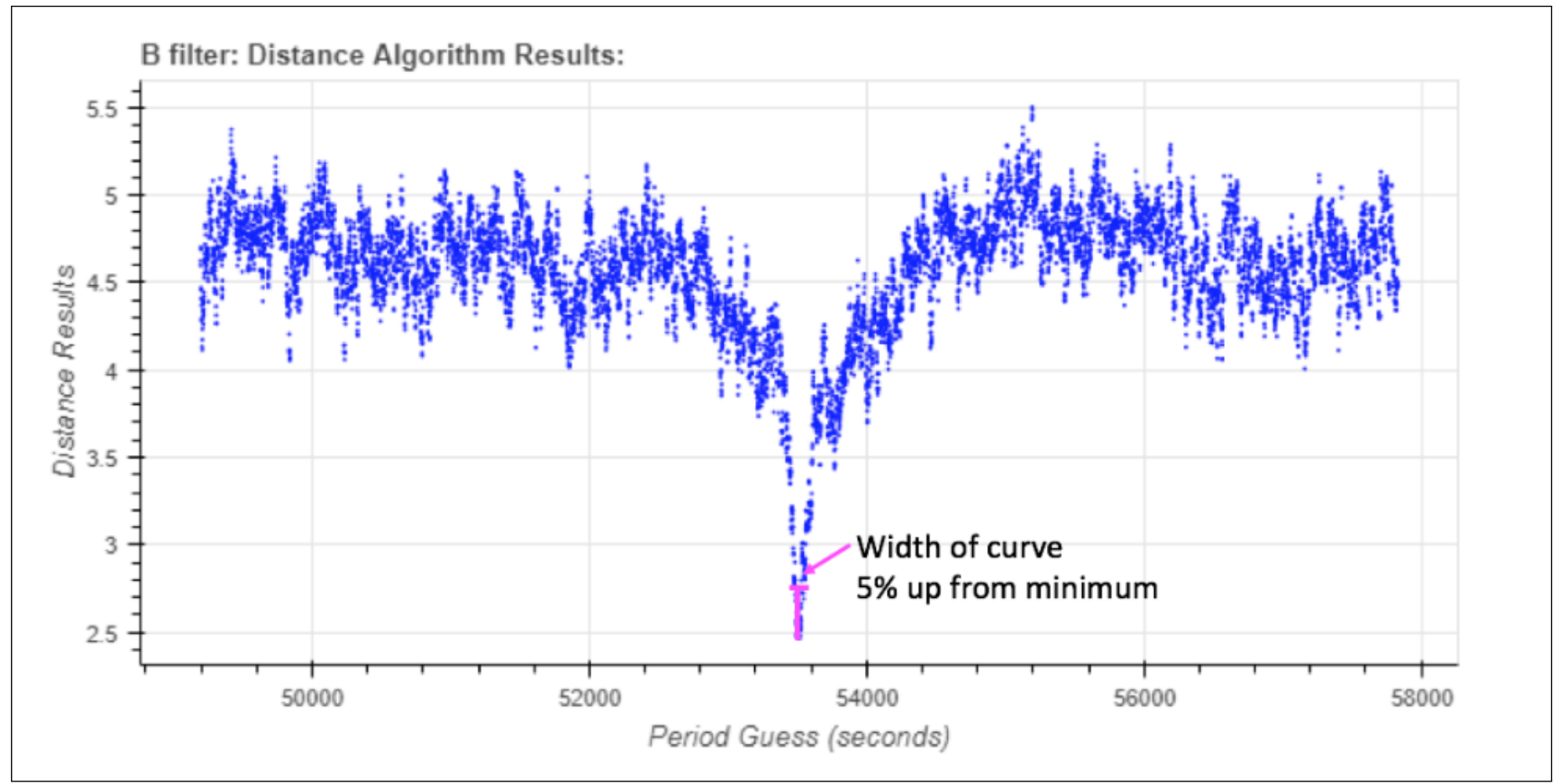

Fig. 13. Method for estimating error of PDM algorithms by finding the width of the curve 5\% of the way up from its minimum value. 


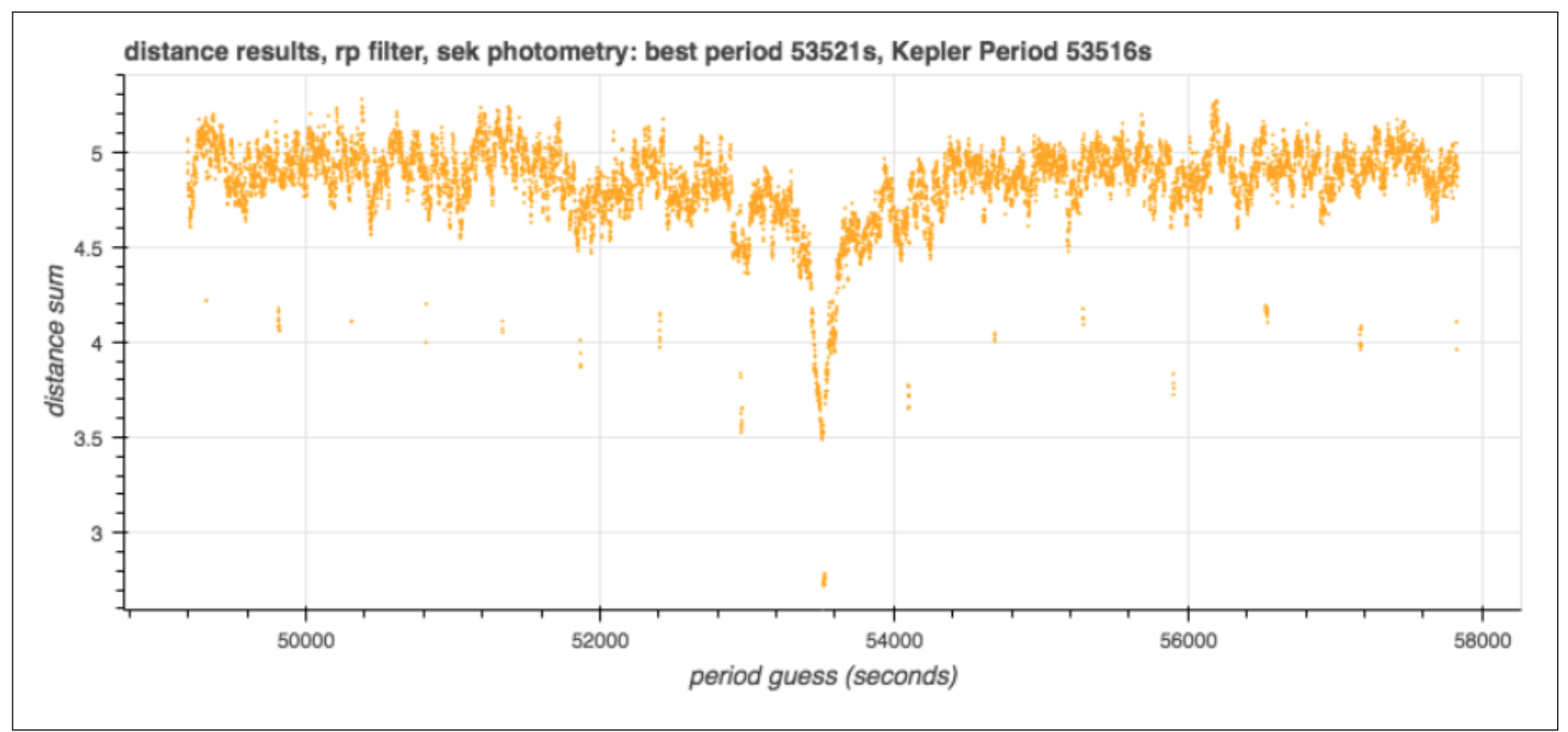

Fig. 14. Primary and secondary minima for distance method on SDSS $r^{\prime}$ filter images with sek photometry.

omy education project. Robotic Telescopes, Student Research and Education Proceedings, 1(1), 221-235.

Fitzgerald, M. e. a. (2018b). The our solar siblings pipeline: Tackling the data issues of the scaling problem for robotic telescope based astronomy education projects. Robotic Telescope, Student Research and Education Proceedings, 1(1), 347358.

Giallongo, E. e. a. (2008). The performance of the blue prime focus large binocular camera at the large binocular telescope. Astronomy and Astrophysics, 482(1), 349-357.

Hensley, H. (n.d.). Period finder simulation. Retrieved from https://www.desmos.com/calculator/ o3thrlvlvg

Kirk, B. e. a. (2016). Kelper eclipsing binary stars. vii. the catalog of eclipsing binaries found in the entire kepler data set. The Astrophysical Journal, 151(3), 22.

Laher, R., Gorjian, V., Rebull, L., J. Masci, F., Fowler, J., Helou, G., ... Law, N. (2012, 07). Aperture photometry tool. Publications of the Astronomical Society of the Pacific.

Lohr, M. e. a. (2012). Period decrease in three superwasp eclipsing binary candidates near the short period limit. Astronomy and Astrophysics, 542.

Matijevic, G. e. a. (2012). Kepler eclipsing binary stars. iii. classification of kepler eclipsing binary light curves with locally linear embedding. The Astronomical Journal, 143(5), 1-6.

Montogomery, M. H., \& Odonoghue, D. (1999). A derivation of the errors for least squares fitting to time series data. Delta Scuti Star Newsletter, 13, 28-32.

Pickles, A. e. a. (2014). Lcogt network observatory operations (Vol. 9149). Retrieved from https://doi.org/10.1117/12.2055215

Rozmus, D. (2010). Eclipsing binary stars. Seminar Files, 2010 - 2011(1), 1-13.

Schechter, P., \& Mateo, M. (1993). Dophot, a ccd photometry program: Description and tests. Publications of the Astronomical Society of the Pacific, 105, 1342-1353.

Schmitt, J. e. a. (2016). Planet hunters. x. searching for nearby neighbors of 75 planet and eclipsing binary candidates from the $\mathrm{k} 2$ kepler extended mission. The Astronomical Journal, 151-159.

Southworth, J. (2012). Eclipsing binary stars: The royal road to stellar astrophysics. In D. H. F. Arenou (Ed.), (Vol. Proceedings of the workshop 'Orbital Couples: Pas de Deux in the Solar System and the Milky Way' (Paris 2012), pp. 5158).

Stellingwerf, R. F. (1978). Period determination using phase dispersion minimization. The Astrophysical Journal, 224, 953-960.

Stetson, P. (1987). Daophot: A computer program for 
crowded-field stellar photometry. Publications of the Astronomical Society of the Pacific, 99, 191-222. 